\title{
Is cognitive performance associated with anxiety and depression in first-episode psychosis?
}

\section{Lindgren, Maija}

2020-02-15

Lindgren , M , Birling , H , Kieseppä , T \& Tuulio-Henriksson , A 2020 , ' Is cognitive performance associated with anxiety and depression in first-episode psychosis? ' , Journal of Affective Disorders , vol. 263 , pp. 221-227 . https://doi.org/10.1016/j.jad.2019.11.161

http://hdl.handle.net/10138/322512

https://doi.org/10.1016/j.jad.2019.11.161

cc_by_nc_nd

acceptedVersion

Downloaded from Helda, University of Helsinki institutional repository.

This is an electronic reprint of the original article.

This reprint may differ from the original in pagination and typographic detail.

Please cite the original version. 
Research paper

\title{
Is cognitive performance associated with anxiety and depression in first- episode psychosis?
}

\author{
Maija Lindgren $^{\mathrm{a}, *}$, Heli Birling ${ }^{\mathrm{b}}$, Tuula Kieseppä ${ }^{\mathrm{a}, \mathrm{c}}$, Annamari Tuulio-Henriksson ${ }^{\mathrm{b}}$ \\ ${ }^{\text {a } M e n t a l ~ H e a l t h ~ U n i t, ~ F i n n i s h ~ I n s t i t u t e ~ f o r ~ H e a l t h ~ a n d ~ W e l f a r e ~(T H L), ~ P O ~ B o x ~ 30, ~ F I N-00271 H e l s i n k i, ~ F i n l a n d ~}$ \\ ${ }^{\mathrm{b}}$ Department of Psychology and Logopedics, Faculty of Medicine, University of Helsinki, Finland \\ ${ }^{\mathrm{c}}$ Department of Psychiatry, University of Helsinki and Helsinki University Hospital, Finland
}

\section{A B S T R A C T}

Background: In first-episode psychosis (FEP), symptoms of anxiety and de-

pression are common. We examined whether cognitive performance is associated with these clinical measures in FEP during a one-year follow-up.

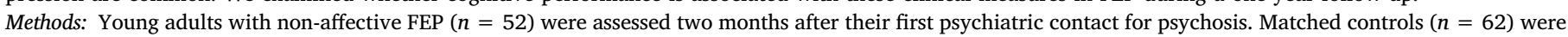

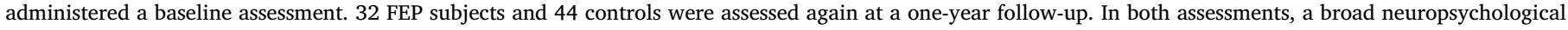

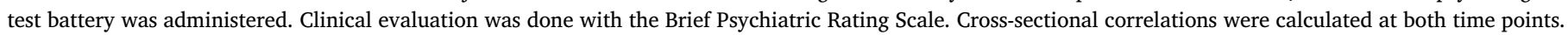
Cognitive test scores were used as independent variables in regression models, predicting both baseline and follow-up symptom levels.

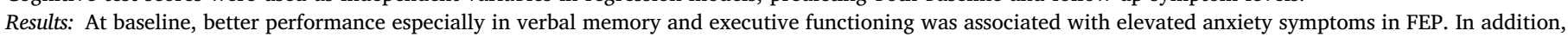

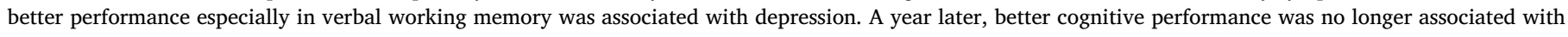
affective symptoms.

Limitations: Small sample sizes are a limitation.

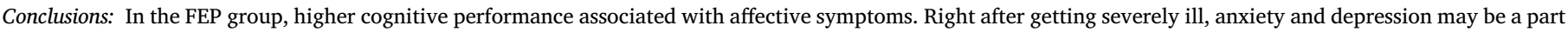

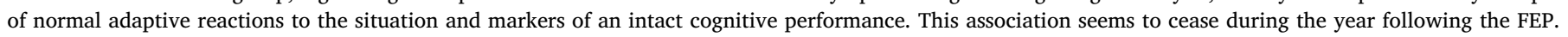

\section{Introduction}

In first-episode psychosis (FEP), a diffuse cognitive decline is often observed (Aas et al., 2014). The decline may be present years before the illness, with a marked increase prior to onset of psychosis (Mesholam-Gately et al., 2009; Mollon and Reichenberg, 2018). In firstepisode schizophrenia, verbal memory along with processing speed is among the cognitive functions with largest deficits (MesholamGately et al., 2009). Moreover, verbal memory may further deteriorate over the course of the illness, while other functions may remain stable after the first episode (Bozikas and Andreou, 2011).

The prevalence of psychiatric comorbidity is common in FEP, anxiety and depressive disorders being among the most common comorbid conditions (Sim et al., 2006). At the symptom level, both anxiety (Buckley et al., 2009; Dernovšek and Šprah, 2009; Hall, 2017) and depression (Coentre et al., 2017; Cotton et al., 2012) are common in individuals with non-affective psychotic disorder, fluctuating across the course of the illness and diminishing with follow-up time and treatment. Factors predisposing to psychosis, such as stress, trauma, or substance abuse, associate with a risk for greater anxiety, and before the first psychotic episode, anxiety may affect cognitive skills and predispose to psychotic interpretations (Hall, 2017). We have previously found that in FEP, negative childhood experiences were associated with anxiety (Lindgren et al., 2017) and with cognitive performance (Morales-Muñoz et al., 2018). A recent review reported the prevalence of clinically significant depressive symptoms after FEP to be $41 \%$ at baseline, and $28 \%$ at one-year (Coentre et al., 2017). Elevated depression predicts poor outcome in FEP (Upthegrove et al., 2014, 2010). On the other hand, better insight - awareness into the illness which is associated with more favorable outcome in many ways, may in fact add risk of more severe levels of depressive symptoms in FEP (Cotton et al., 2012; Drake et al., 2004; Klaas et al., 2017).

Clinical symptoms contribute to the cognitive performance. In the general population, anxiety and depression are associated with lower cognitive performance (Castaneda et al., 2008), especially when viewed at the $g$ factor level, the highest level in the hierarchy of cognitive abilities (Salthouse, 2012). Individuals with major depressive disorder (MDD) show deficits especially in psychomotor speed and memory (Lee et al., 2012). Anxiety and depressive symptoms can affect cognitive performance through impairing attentional control. However, when processing negative information, affective symptoms may increase attention. Compensating the impairments with enhanced effort

\footnotetext{
* Corresponding author.

E-mail address: maija.lindgren@thl.fi (M. Lindgren).
} 
can minimize the effects on performance (Eysenck et al., 2007; Gotlib, Joormann, 2010). While elevated depression seems to be associated with weaker cognitive performance in a linear way, there appears to be an inverted U-relation between anxiety and cognitive performance so that intermediate levels of anxiety relate to best cognitive performance in the general population (Salthouse, 2012).

The association between cognitive performance and affective symptoms in the context of FEP - and in psychotic disorders in general has been studied only sparsely and the results have been inconsistent. Cognitive impairment may be associated with using maladaptive coping strategies, such as avoidance and withdrawal, in a stressful situation like when diagnosed with a severe mental illness (Lysaker et al., 2005). In one study anxiety and depressive symptoms in FEP were associated with lower verbal processing speed but not with memory and planning functions or general neurocognitive performance (Stouten et al., 2017). In previous studies on schizophrenia samples, anxiety has not been found to associate with cognitive performance (Aikawa et al., 2018; Buonocore et al., 2018). In one study, depression was found to be associated with decreased cognitive speed but not motor speed or working memory in schizophrenia (Brébion et al., 2009), but in FEP, no association between neuropsychological functioning and depressive symptoms has been detected (Lucas et al., 2004; Ohmuro et al., 2015). A systematic review found no association between neurocognition and depression related to lifetime occurrence of psychosis (Dominguez et al., 2009). One study explored the trajectories in cognition over two years in FEP and found that a subgroup with cognitive deterioration over time had more depressive symptoms, linking clinical prognosis to outcomes in cognition (Sánchez-Torres et al., 2018). Impairment in social cognition may be associated with negative symptoms in FEP (Healey et al., 2016), but less is known of the association of social cognition with anxiety or depression. Age can affect the association between cognitive performance and affective symptoms (Potvin et al., 2013). Moreover, gender differences may exist: in individuals with schizophrenia, verbal recall has been found to be inversely affected by depression in women and anxiety in men (Brébion et al., 2013).

In a recent study, depressive symptoms of young people with FEP were associated with higher cognitive performance, especially processing speed (Herniman et al., 2018). Furthermore, a recent meta-analysis reported significantly higher comorbidity prevalence rate for social anxiety disorder in psychotic disorders in outpatients compared to inpatients, leading to a discussion about the possible effect of greater insight and awareness of anxiety symptoms (McEnery et al., 2019). These results indicate that it is possible that in people with good insight - associated with higher cognitive capacity (Aleman et al., 2006; Rajji et al., 2014) - anxiety and depression may develop as a reaction to the difficult experience of getting ill and the possible stigma related to that (Birchwood et al., 2007). Targeting comorbid affective symptoms represents an important treatment strategy (Hall, 2017). Understanding how cognitive performance and clinical features of the FEP are linked may have clinical implications for intervention strategies of early psychosis.

The present study set out to explore whether neurocognitive functioning and social cognition are associated with anxiety and depressive symptoms among young adults with FEP and matched population controls. Based on previous literature, either higher or lower cognitive resources could be hypothesized to be associated with affective symptoms in FEP. These questions were investigated at two phases: soon after the onset of treatment for FEP and one year after.

\section{Methods}

\subsection{Participants and study protocol}

The FEP group consisted of patients participating in the Helsinki Psychosis Study. These individuals, aged 18 to 40 , had their first psychiatric treatment contact for psychosis in hospitals and outpatient clinics of the City of Helsinki and Helsinki University Hospital between December 2010 and July 2016. The participants were interviewed with Brief Psychiatric Rating Scale, Expanded version 4.0, BPRS (Ventura et al., 1993), and as a criterion for inclusion, psychosis was defined as a score $\geq 4$ (moderate or higher) in unusual thought content (delusions) or hallucinations. Exclusion criteria were psychotic disorders that were substance-induced or caused by a general medical condition. Participants with affective psychosis (schizoaffective disorder, bipolar I disorder, or major depressive disorder with psychotic features) were excluded from this study, as affective symptoms have a different meaning in these disorders than in nonaffective psychotic disorders. The study protocol included one meeting as soon as possible after the treatment had started and the patients were able to give consent to participate as judged by the treating personnel. We utilized data from 1) the two-month assessment (when the cognitive testing was done, in order to avoid testing in the most acute phase of the illness; referred here as baseline assessment) and 2) the one-year follow-up.

Age- and gender-matched control participants from the same catchment area were recruited from the Finnish Population Information System. Psychotic disorders were an exclusion criterion, as were any conditions preventing MRI, and chronic neurological or endocrinological diseases. Other mental health problems were allowed. The controls were assessed at baseline and again one year later, with the same measures as the patients.

The study protocol was carried out in accordance with the Declaration of Helsinki. All the participants gave written informed consent to participation. The study protocol was approved by the Ethics Committee of the Hospital District of Helsinki and Uusimaa, and by the institutional review boards of the Finnish Institute for Health and Welfare, Helsinki, and the University of Helsinki.

\subsection{Measures}

\subsubsection{Cognitive testing}

Cognitive tests were administered to both patients and controls by a psychologist at both time points. As the same psychologist usually conducted both the interview and the testing, there was no possibility to be blinded towards the diagnoses.

The test battery comprised the Wechsler Adult Intelligence Scale (Block Design, Vocabulary, Digit Symbol), Wechsler Memory Scale (Logical Memory, Letter-Number Sequencing, Spatial Span, Word List, Visual Reproduction), Trail Making Test, Verbal Fluency, Tapping Task, and The Continuous Performance Test, Identical Pairs (CPT-IP). Social cognition was measured with the Hinting Task.

The FEP participants were tested with a slightly broader battery compared to the controls, and the baseline testing was broader than the one-year testing. All test methods administered to the controls were included in the test battery of the patients. The cognitive variables used can be seen in Supplementary Table 1.

\subsubsection{Clinical measures}

Anxiety and depression were assessed both at baseline and at oneyear with the 24-item version of BPRS (Ventura et al., 1993) on a scale of 1-7, with higher score indicating more severe symptoms. Positive psychotic symptoms were calculated as the mean of BPRS current hallucinations, unusual thought content, and conceptual disorganization item scores. Trained research staff (psychologists and nurses) conducted the interviews.

Insight was measured in the FEP group at both study phases using the Schedule for the Assessment of Insight, Expanded version (SAI-E), consisting of items assessing insight and compliance to treatment (Kemp and David, 1996). The sum scale of the insight items was used in the analyses. Level of functioning of all participants was assessed with the Global Assessment of Psychosocial Functioning (GAF) (American Psychiatric Association, 2013) on a scale of 0-100 in each study phase. 
Diagnoses were set by a senior psychiatrist based on all available information, including the Structured Clinical Interview for the DSMIV, Research Version (SCID-I/P) (First et al., 2002) as well as medical records from received mental health treatment. Information on medication was collected from interviews and medical records. Use of antidepressant (citalopram, escitalopram, fluoxetine, paroxetine, sertraline, venlafaxine, or mirtazapine) was coded as yes/no. Chlorpromazine equivalent was calculated from antipsychotic medication.

\subsubsection{Analyses}

A single exploratory factor model of the neurocognitive variables was formed to summarize cognitive performance. This $g$ factor solution was done for both baseline (Supplementary Table 2, Lindgren et al., 2018) as well as the one-year testing data (Supplementary Table 3).

All further analyses employed IBM SPSS Statistics for Windows, version 25. First, an inverted square root transformation was used to normalize the scores of Logical Memory delayed recognition and Hinting Task. Trail Making and Tapping Task performance times were transformed to denote speed, by taking the reciprocal, normalizing distributions and making higher values indicate better performance also in these tasks. Other cognitive variables were used as raw scores. Logarithmic transformations were made to BPRS anxiety and depression scores to be used in the regression models.

Patient and control groups were compared with Pearson's ChiSquare or the Mann-Whitney $U$-test. Spearman correlations were used to examine the associations between cognitive performance and anxiety and depression, calculated separately for the FEP and control groups. These cross-sectional correlations were calculated separately at both time points, to see whether any associations would replicate, and longitudinally between baseline cognitive variables and one-year clinical variables.

Then, linear regression models were computed for the FEP group, with anxiety or depression as the dependent variable in separate models. The independent variables in the regression models were cognitive variables one-by-one, selecting those variables with significant correlations with anxiety and depression. This was done both crosssectionally for the two time points and longitudinally to predict oneyear clinical measures with baseline cognition. We first separately tested which of the background and clinical variables were the significant predictors: age, gender, years of education, GAF functioning score, positive psychotic symptoms, antipsychotic medication, and insight. We then included those with significance in the final regression models as covariates. Standardized $\beta$ values are given to allow comparability between models.

\section{Results}

\subsection{Baseline}

52 non-affective FEP and 62 control participants were included in the study at the baseline phase (Table 1). The FEP group and the controls did not differ in age or gender. Of the FEP group, $26(50.0 \%)$ participants had schizophrenia, 11 (21.2\%) had schizophreniform disorder, $12(23.1 \%)$ had psychotic disorder NOS, and $3(5.8 \%)$ had brief psychotic disorder. At the time of the study enrollment, $30(57.7 \%)$ of the FEP group were inpatients. Of the FEP group, 16 (30.8\%) were in remission during the past week according to criteria by Andreasen et al. (2005), while the rest of the group were currently experiencing psychotic level symptoms.

At the baseline assessment, all but three FEP participants (5.8\%) were prescribed with antipsychotic medication. In addition, 14 (26.9\%) patients were prescribed with antidepressants. FEP participants with antidepressant at baseline showed higher baseline cognitive performance than the other patients, as assessed with $g$ factor (Mann-Whitney $U=362.0, p=.048$ ).

At baseline, FEP participants had elevated anxiety and depression and they performed worse on the cognitive tasks at the group level than the controls (Table 1). Age of the FEP participants was not correlated with affective symptoms, and there were no gender differences in anxiety or depression. In the FEP group, the scores of anxiety and depression were correlated $(r=0.63, p<.001)$. Insight, as measured with SAI-E, did not correlate significantly with depression, anxiety, or $g$ factor at baseline. Of the individual tasks, higher performance in Trail Making B $(r=0.37, p=.010)$ and CPT-IP $(r=0.46, p=.001)$ correlated with better insight.

Spearman correlations between the cognitive tasks and clinical measures at baseline are shown in Table 2. In FEP, anxiety correlated positively with the $g$ factor and at the task level, with WMS-III Logical memory immediate and recognition, as well as with Trail Making B. Depressive symptoms of the FEP correlated positively with the $g$ factor and with Letter-Number Sequencing. In controls, cognition did not correlate with anxiety and depression (Table 2).

In regression models, anxiety and depression symptom levels in the FEP group were predicted by the cognitive variables that were significantly correlated with the symptoms. Of the background and clinical variables, positive psychotic symptoms predicted anxiety $(\beta=0.4$, $p=.003$ ), whereas age, gender, education, level of functioning, antipsychotic medication (chlorpromazine equivalent), or insight were not significant predictors of anxiety. In the final models, we therefore adjusted only for positive symptoms. Of the cognitive predictors, $g$ factor $(\beta=0.3, p=.037)$, Logical Memory delayed recognition $(\beta=0.3$, $p=.008)$, and Trail Making B $(\beta=0.4, p=.007)$ significantly predicted anxiety in the FEP group, whereas Logical Memory immediate just fell off the significance level $(\beta=0.3, p=.051)$.

None of the background and clinical variables predicted depression. Of the cognitive variables, $g$ factor did not significantly predict depression $(\beta=0.2, p=.086)$, but Letter-Number Sequencing did $(\beta=0.3, p=.016)$.

\subsection{One-year follow-up}

Information on both cognitive functioning and symptoms at oneyear was available for 32 individuals with FEP and 44 controls (Table 1). FEP participants attending the one-year follow-up did not differ on age, education, baseline depression, anxiety, antipsychotic medication, or $g$ factor from those not attending, but their baseline functioning (mean 45.0 vs. $39.8, U=450.0, p=.035$ ) and insight (mean 17.4 vs. $13.3, U=432.5, p=.017$ ) was higher.

One year after entering treatment, $17(53.1 \%)$ of the FEP group were in remission. $23(71.9 \%)$ of the FEP group were still prescribed with antipsychotic medication and $11 \mathrm{FEP}$ and 4 control individuals were prescribed with antidepressants at one-year.

At one-year, the FEP participants still reported more severe anxiety and depression and performed worse on the cognitive tasks than the controls (Table 1). In FEP, insight at one-year was not significantly correlated with anxiety or depression. Insight correlated significantly with one-year $g$ factor $(r=0.67, p<.001)$ and at the task level, with Digit Symbol $(r=0.44, p=.021)$ and Tapping, dominant hand $(r=-0.40, p=.044)$.

Spearman correlations between the one-year cognitive tasks and anxiety and depression at one-year can be seen in Supplementary Table 4. At this phase, there were no significant correlations in either group, and therefore we did not perform regression models.

Correlations were finally calculated between cognitive variables at baseline and affective variables at one-year follow-up (Supplementary Table 5), with no significant correlations in the FEP group, thus regression models were not performed.

\section{Discussion}

The aim of the present study was to investigate whether cognitive performance and anxiety and depressive symptoms were associated 
Table 1

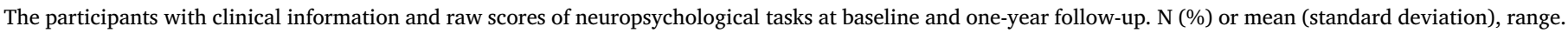

\begin{tabular}{|c|c|c|c|c|c|c|}
\hline & $\begin{array}{l}\text { Baseline } \\
\text { FEP, } n=52\end{array}$ & Controls, $n=62$ & Group difference $^{a}$ & $\begin{array}{l}\text { One-year follow-up } \\
\text { FEP, } n=32\end{array}$ & Controls, $n=44$ & Group difference $^{\mathrm{a}}$ \\
\hline Age & 26.7 (5.7), 18.4-41.3 & 26.3 (5.9), 19.1-43.8 & ns & - & - & - \\
\hline Female & $21(40.4 \%)$ & $21(33.9 \%)$ & $\mathrm{ns}$ & $14(43.8 \%)$ & $11(25.0 \%)$ & ns \\
\hline Education $^{\mathrm{b}}$ & 14.0 (3.4), 9.5-23.5 & 14.8 (2.4), $11.0-22.0$ & $p=.029$ & - & - & - \\
\hline GAF & 42.6 (10.2), 25-75 & 83.2 (9.0), 55-95 & $p<.001$ & 53.7 (12.6), 30-70 & $\begin{array}{l}82.1(10.4) \\
40-96\end{array}$ & $p<.001$ \\
\hline $\begin{array}{l}\text { BPRS mean of current positive } \\
\text { symptoms }\end{array}$ & $2.1(1.2), 1.0-5.7$ & $1.0(0.1), 1.0-1.7$ & $p<.001$ & $1.3(0.4), 1.0-2.7$ & $1.0(0.1), 1.0-1.3$ & $p=.002$ \\
\hline BPRS depression & 2.4 (1.5), $1-6$ & $1.2(0.5), 1-3$ & $p<.001$ & $1.5(0.9), 1-4$ & $1.2(0.6), 1-4$ & $p=.018$ \\
\hline BPRS anxiety & 2.9 (1.7), 1-7 & $1.6(1.1), 1-5$ & $p<.001$ & $2.2(1.3), 1-5$ & $1.6(1.0), 1-6$ & $p=.020$ \\
\hline SAI-E & 15.5 (6.5), $0-24$ & - & - & 18.1 (4.5), $6-24$ & - & - \\
\hline$g$ factor & $-0.5(0.9),-2.4-1.6$ & $0.5(0.8),-1.4-2.9$ & $p<.001$ & $-0.3(1.0),-2.4-1.9$ & $\begin{array}{l}0.4(0.8) \\
-1.0-2.5\end{array}$ & $p=.004$ \\
\hline \multicolumn{7}{|l|}{ WAIS-III tests: } \\
\hline Block Design & 45.5 (12.4), 12-65 & 50.9 (12.3), 11-68 & $p=.018$ & 48.8 (12.7), 12-63 & - & - \\
\hline Vocabulary & 44.6 (10.5), $22-64$ & 48.7 (9.6), 22-64 & $p=.037$ & 48.0 (10.6), 18-64 & - & - \\
\hline Digit Symbol & 64.0 (15.1), 32-105 & 79.9 (12.7), 54-111 & $p<.001$ & 71.4 (19.2), 39-110 & $\begin{array}{l}83.1(11.2) \\
58-112\end{array}$ & $p=.001$ \\
\hline \multicolumn{7}{|l|}{ WMS-III tests: } \\
\hline Logical Memory, immediate & 36.4 (11.6), 3-62 & 45.1 (9.7), 17-63 & $p<.001$ & 41.2 (9.9), 25-64 & 45.5 (8.5), 24-61 & $p=.049$ \\
\hline Logical Memory, delayed & 22.2 (9.1), 4-42 & 28.9 (7.0), $12-44$ & $p<.001$ & 26.5 (8.4), 13-45 & $29.6(6.3), 16-40$ & ns \\
\hline Logical Memory, delayed recognition & 25.4 (3.0), 17-30 & 26.6 (2.3), $17-30$ & $p=.026$ & 26.0 (2.6), 19-30 & - & - \\
\hline Letter-Number Sequencing & 9.4 (2.4), 5-17 & 10.8 (2.3), 6-18 & $p=.002$ & $9.6(2.4), 6-14$ & 10.9 (2.9), 5-18 & ns \\
\hline Spatial Span & 18.0 (3.5), $10-26$ & $19.3(2.6), 13-26$ & $p=.015$ & 18.4 (3.3), 9-25 & - & - \\
\hline Word List, immediate & $30.7(6.0), 17-44$ & - & - & - & & - \\
\hline Word List, long delay & $6.6(3.4), 0-12$ & - & - & - & & - \\
\hline Word List, delayed recognition & 22.7 (1.6), 19-24 & - & - & - & & - \\
\hline Visual Reproduction, immediate & 90.6 (13.4), 31-103 & - & - & - & & - \\
\hline Visual Reproduction, delayed & 74.5 (22.7), $15-103$ & - & - & - & & - \\
\hline Hinting Task & $15.8(3.1), 4-20$ & 17.4 (1.6), $13-20$ & $p=.003$ & - & & - \\
\hline Trail Making A (s) & 34.3 (18.1), 13-118 & $24.2(6.3), 13-40$ & $p<.001$ & 31.0 (12.3), 15-61 & $20.8(6.3), 13-42$ & $p<.001$ \\
\hline Trail Making B (s) & 71.7 (25.8), 33-164 & 49.0 (16.9), 22-132 & $p<.001$ & 63.3 (24.4), 19-141 & $\begin{array}{l}49.6(23.7) \\
27-155\end{array}$ & $p=.001$ \\
\hline Verbal Fluency & 48.2 (13.4), 20-89 & 59.5 (13.9), 40-99 & $p<.001$ & 47.6 (15.5), 18-74 & $\begin{array}{l}61.8(14.0) \\
31-94\end{array}$ & $p=.001$ \\
\hline CPT-IP D-prime & 2.40 (0.67), $0.73-3.85$ & $2.8(0.5), 1.5-3.7$ & $p=.001$ & $2.6(0.7), 1.2-3.7$ & $3.0(0.6), 1.8-4.0$ & $p=.031$ \\
\hline Tapping dominant hand (ms) & $\begin{array}{l}178.4(23.3) \\
129.6-235.6\end{array}$ & $\begin{array}{l}166.0(16.1) \\
119.2-213.1\end{array}$ & $p=.003$ & $\begin{array}{l}186.0(26.9) \\
135.5-243.1\end{array}$ & - & - \\
\hline Tapping nondominant hand (ms) & $\begin{array}{l}215.5(36.7) \\
152.4-334.1\end{array}$ & $\begin{array}{l}197.1(28.3) \\
134.7-302.3\end{array}$ & $p=.002$ & $\begin{array}{l}224.7 \text { (39.9), } \\
179.3-347.1\end{array}$ & - & - \\
\hline
\end{tabular}

a Pearson's Chi-Square or Mann-Whitney $U$ test.

b Full-time education in years, self-reported

FEP, first-episode psychosis

GAF, Global Assessment of Psychosocial Functioning

BPRS, Brief Psychiatric Rating Scale, Expanded version

CPT-IP, Continuous Performance Test - Identical Pairs

SAI-E, Schedule for the Assessment of Insight, Expanded version.

among FEP participants, both at the beginning of treatment and at oneyear follow-up, and to compare FEP participants with controls at baseline and follow-up. Not surprisingly, individuals with FEP reported more anxiety and depression compared to the controls. However, there was variation in the FEP group, and many of the patients were comparable to the controls in terms of affective symptoms, reflecting heterogeneity in clinical measures among the FEP group. Both anxiety and depressive symptoms of the FEP participants correlated positively with the global cognitive index at baseline, indicating that these symptoms were associated with higher cognitive performance at the beginning of treatment. Verbal long-term memory and executive functioning were associated with anxiety. In the regression models, higher performance in two separate tasks - Logical Memory delayed recognition, assessing verbal memory retrieval, and Trail Making B, assessing executive functioning - predicted elevated anxiety, independently of positive psychotic symptoms. FEP participants with more depressive symptoms scored better particularly in Letter-Number Sequencing, a measure of verbal working memory.

After one year of the initiation of treatment, the correlations disappeared, and neither the cross-sectional associations nor associations between baseline cognition with one-year clinical measures were significant. At one-year, anxiety and depression were milder compared to the early stage of illness. The antidepressive treatment during the oneyear follow-up had probably relieved the affective symptoms, which might have diminished the association of those symptoms with cognitive performance. Concerning received treatment, antidepressant medication at baseline was associated with higher cognitive performance in the FEP group, which was an interesting finding outside the main scope of the current study. Previously, antidepressants have been shown to advantage cognition in MDD (Keefe et al., 2014) and slightly also in schizophrenia, especially executive functioning and a composite cognition score (Vernon et al., 2014).

The correlation between anxiety and depression with cognitive performance was in the opposite direction among the controls than in 
Table 2

Spearman correlations between the baseline cognitive tasks and anxiety and depression at baseline. Significant correlations are followed with $p$-value.

\begin{tabular}{|c|c|c|c|c|}
\hline & \multicolumn{2}{|l|}{ FEP } & \multicolumn{2}{|l|}{ Controls } \\
\hline & BPRS Anxiety, $n=52$ & BPRS Depression, $n=52$ & BPRS Anxiety, $n=65$ & BPRS Depression, $n=65$ \\
\hline$g$ factor & $.30(p=.031)$ & $.30(p=.030)$ & -0.12 & -0.10 \\
\hline \multicolumn{5}{|l|}{ WAIS-III tests: } \\
\hline Block Design & -0.09 & -0.01 & -0.10 & -0.06 \\
\hline Vocabulary & -0.03 & .11 & .00 & .00 \\
\hline Digit Symbol & .24 & .22 & -0.03 & .14 \\
\hline \multicolumn{5}{|l|}{ WMS-III tests: } \\
\hline Logical Memory, immediate & $.32(p=.027)$ & .19 & -0.25 & -0.20 \\
\hline Logical Memory, delayed & .26 & .15 & -0.22 & -0.21 \\
\hline Logical Memory, delayed recognition & $.39(p=.007)$ & .26 & -0.07 & -0.07 \\
\hline Letter-Number Sequencing & .18 & $.28(p=.049)$ & -0.03 & -0.17 \\
\hline Spatial Span & .09 & .08 & .03 & .05 \\
\hline Word List, immediate & .20 & .10 & - & - \\
\hline Word List, long delay & .10 & .08 & - & - \\
\hline Word List, delayed recognition & .21 & .15 & - & - \\
\hline Visual Reproduction, immediate & .06 & .13 & - & - \\
\hline Visual Reproduction, delayed & .03 & .09 & - & - \\
\hline Hinting Task & -0.05 & .10 & .00 & .10 \\
\hline Trail Making A & .17 & .18 & -0.20 & -0.01 \\
\hline Trail Making B & $.39(p=.006)$ & .22 & -0.13 & -0.11 \\
\hline Verbal Fluency & .02 & .05 & .02 & -0.06 \\
\hline CPT-IP mean & .05 & .12 & .12 & .17 \\
\hline Tapping, dominant hand & -0.28 & -0.09 & .03 & -0.07 \\
\hline Tapping, nondominant hand & -0.24 & -0.24 & -0.11 & -0.21 \\
\hline
\end{tabular}

FEP, first-episode psychosis.

BPRS, Brief Psychiatric Rating Scale, Expanded version.

CPT-IP, Continuous Performance Test - Identical Pairs.

the FEP group. The affective symptoms of the controls associated with worse cognitive performance, although the correlations did not reach statistical significance. This is in line with previous results of anxiety and depression associating with cognitive decline in the general population (Castaneda et al., 2008; Eysenck et al., 2007; Salthouse, 2012). According to a previous study, the association between cognitive performance and anxiety may be shaped like an inverted $U$ so that the highest cognitive performance is associated with intermediate levels of anxiety (Salthouse, 2012). In the current study, the FEP group showed significantly higher affective symptom levels than the controls. The differences in the association between cognition and symptoms between groups could reflect the different positions of the groups on this U-shape curve. During the follow-up, the patients might have approached controls in this sense while their anxiety symptoms reduced.

Dysregulation of emotions in FEP has been linked with cognitive decline in some studies (Stouten et al., 2017) and with unimpaired cognitive performance in other studies (Herniman et al., 2018). Our results are in line with a hypothesis that high cognitive resources may associate with manifestation of affective symptoms, which may be an adequate reaction on the situation of getting severely ill (Herniman et al., 2018). According to this hypothesis, high cognitive resources may lead to better understanding of the situation after FEP, which can lead to a depressive reaction, whereas lack of these reactive symptoms may indicate lower insight (Herniman et al., 2018). However, in the present study, insight did not explain severity of anxiety or depression. Moreover, whereas insight was not correlated with the $g$ factor at baseline, the association was significant a year after the onset of treatment. However, FEP patients attending the follow-up assessment had better insight and higher functioning at baseline, thus limiting conclusions to be drawn on the one-year results concerning insight.

\subsection{Strengths and limitations}

Strengths of this study include its longitudinal design, allowing for cross-sectional analyses at two time points and investigating longitudinal associations. Some cognitive tests were administered only to the FEP group and not to controls, and some only at baseline and not at follow-up, thus reducing the possibility to conduct all longitudinal analyses. The symptom levels of the FEP participants were first assessed soon after the initiation of treatment, offering information at the clinical state of still having psychotic symptoms. Affective psychoses were not included in the study, thus the results tell only of FEP patients on the schizophrenia spectrum. The controls were allowed to have other psychiatric problems than psychosis, and about a fifth had a lifetime psychiatric diagnosis, most usually depression. However, current anxiety and depressive symptoms were mild in the control group. Some of the FEP participants also had a diagnosis of mood or anxiety disorder, but the present study included only symptom level analysis using the BPRS interview (Ventura et al., 1993). The relationship of affective symptoms and cognition among FEP patients should be further studied using also specific symptom scales.

We took into account a number of background and clinical variables, such as positive symptom level and antipsychotic medication. However, many factors possibly associating with cognitive performance were not available in the current study. For example longer DUP may be associated with more substantial cognitive deficits (Wang et al., 2016), of which we had no data. Moreover, we were not able to evaluate the premorbid cognitive level of the FEP participants.

Finally, in relation to the number of comparisons made, our sample size was small because of the extensive overall study protocol in the Helsinki Psychosis Study, including also laboratory assessments and MRI and fMRI at several time points, not used in the present study. Some participants were lost at the one-year follow-up, the reasons varying from refusal to not reaching the participant. Due to the explorative nature of our study, we did not correct for multicomparison.

\section{Conclusions}

The results of the present study suggest that anxiety and depression may be markers of higher cognitive performance at the early stage of psychosis. Affective symptoms may be considered important to be taken into account at service entry. Anxiety and depression are distressing symptoms affecting everyday life, even after the positive psychotic symptoms have disappeared, and their association with cognitive 
performance right after getting ill may be clinically important.

\section{Role of the funding source}

This study was funded by the European Union's Seventh Framework Programme for project METSY-Neuroimaging platform for characterization of metabolic co-morbidities in psychotic disorders (\#602478), the Yrjö Jahnsson Foundation (\#6979 to ML), the Sigrid Juselius Foundation, The Juho Vainio Foundation (ML), and the Jalmari and Rauha Ahokas Foundation (ML). The funding organizations played no further part in study design, in the collection, analysis and interpretation of data, or in writing of the paper.

\section{CRediT authorship contribution statement}

Maija Lindgren: Methodology, Formal analysis, Writing - original draft, Writing - review \& editing, Visualization. Heli Birling: Methodology, Formal analysis, Writing - review \& editing. Tuula Kieseppä: Conceptualization, Writing - review \& editing. Annamari Tuulio-Henriksson: Methodology, Writing - review \& editing.

\section{Declaration of Competing Interest}

ML has received honoria from Lundbeck. All other authors declare that they have no conflicts of interest.

\section{Acknowledgments}

Helsinki Psychosis Study is a collaboration between the Finnish Institute for Health and Welfare, University Hospital District of Helsinki and Uusimaa, and Aalto University. The authors wish to thank Jaana Suvisaari, Marjut Grainger, and the whole study group, as well as all the participants in the study.

\section{Supplementary materials}

Supplementary material associated with this article can be found, in the online version, at doi:10.1016/j.jad.2019.11.161.

\section{References}

Aas, M., Dazzan, P., Mondelli, V., Melle, I., Murray, R.M., Pariante, C.M., 2014. A systematic review of cognitive function in first-episode psychosis, including a discussion on childhood trauma, stress, and inflammation. Front. Psychiatry 8, 182.

Aikawa, S., Kobayashi, H., Nemoto, T., Matsuo, S., Wada, Y., Mamiya, N., Yamaguchi, T., Katagiri, N., Tsujino, N., Mizuno, M., 2018. Social anxiety and risk factors in patients with schizophrenia: relationship with duration of untreated psychosis. Psychiatry Res. 263, 94-100.

Aleman, A., Agrawal, N., Morgan, K.D., David, A.S., 2006. Insight in psychosis and neuropsychological function: meta-analysis. Br. J. Psychiatry 189, 204-212 Sep.

American Psychiatric Association, 2013. Diagnostic and Statistical Manual of Mental Disorders. American Psychiatric Publishing, Arlington, VA.

Andreasen, N.C., Carpenter, W.T.Jr, Kane, J.M., Lasser, R.A., Marder, S.R., Weinberger, D.R., 2005. Remission in schizophrenia: proposed criteria and rationale for consensus. Am. J. Psychiatry 162 (3), 441-449 Mar.

Birchwood, M., Trower, P., Brunet, K., Gilbert, P., Iqbal, Z., Jackson, C., 2007. Social anxiety and the shame of psychosis: a study in first episode psychosis. Behav. Res. Ther. 45, 1025-1037.

Bozikas, V.P., Andreou, C., 2011. Longitudinal studies of cognition in first episode psychosis: a systematic review of the literature. Aust. N. Z. J. Psychiatry 45, 93-108.

Brébion, G., David, A.S., Jones, H.M., Pilowsky, L.S., 2009. Working memory span and motor and cognitive speed in schizophrenia. Cognit. Behav. Neurol. 22, 101-108.

Brébion, G., Villalta-Gil, V., Autonell, J., Cervilla, J., Dolz, M., Foix, A., Haro, J.M., Usall, J., Vilaplana, M., Ochoa, S., 2013. Cognitive correlates of verbal memory and verbal fluency in schizophrenia, and differential effects of various clinical symptoms between male and female patients. Schizophr. Res. 147, 81-85.

Buckley, P.F., Miller, B.J., Lehrer, D.S., Castle, D.J., 2009. Psychiatric comorbidities and schizophrenia. Schizophr. Bull. 35, 383-402.

Buonocore, M., Bosia, M., Baraldi, M.A., Bechi, M., Spangaro, M., Cocchi, F., Bianchi, L., Guglielmino, C., Mastromatteo, A.R., Cavallaro, R., 2018. Exploring anxiety in schizophrenia: new light on a hidden figure. Psychiatry Res. 268, 312-316.

Castaneda, A.E., Tuulio-Henriksson, A., Marttunen, M., Suvisaari, J., Lönnqvist, J., 2008. A review on cognitive impairments in depressive and anxiety disorders with a focus on young adults. J. Affect Disord. 106, 1-27.

Coentre, R., Talina, M.C., Góis, C., Figueira, M.L., 2017. Depressive symptoms and suicidal behavior after first-episode psychosis: a comprehensive systematic review. Psychiatry Res. 253, 240-248 Jul.

Cotton, S.M., Lambert, M., Schimmelmann, B.G., Mackinnon, A., Gleeson, J.F.M., Berk, M., Hides, L., Chanen, A., McGorry, P.D., Conus, P., 2012. Depressive symptoms in first episode schizophrenia spectrum disorder. Schizophr. Res. 134, 20-26.

Dernovšek, M.Z., Šprah, L., 2009. Comorbid anxiety in patients with psychosis. Psychiatr. Danub. 21 (Suppl 1), 43-50 Sep.

Dominguez, M.D.G., Viechtbauer, W., Simons, C.J., van Os, J., Krabbendam, L., 2009. Are psychotic psychopathology and neurocognition orthogonal? a systematic review of their associations. Psychol. Bull. 135, 157-171.

Drake, R.J., Pickles, A., Bentall, R.P., Kinderman, P., Haddock, G., Tarrier, N., Lewis, S.W., 2004. The evolution of insight, paranoia and depression during early schizophrenia. Psychol. Med. 34, 285-292.

Eysenck, M.W., Derakshan, N., Santos, R., Calvo, M.G., 2007. Anxiety and cognitive performance: attentional control theory. Emotion 7 (2), 336-353 May.

First, M.B., Spitzer, R.L., Gibbon, M., Williams, J.B.W., 2002. Structured clinical interview for dsm-iv-tr axis i disorders, research version, patient edition. (SCID-I/P). Biometrics Research. New York State Psychiatric Institute, New York, NY Revision: January 2007.

Gotlib, I.H., Joormann, J., 2010. Cognition and depression: current status and future directions. Annu. Rev. Clin. Psychol. 6, 285-312.

Hall, J., 2017. Schizophrenia - an anxiety disorder? Br. J. Psychiatry 211 (5), 262-263 Nov.

Healey, K.M., Bartholomeusz, C.F., Penn, D.L., 2016. Deficits in social cognition in first episode psychosis: a review of the literature. Clin. Psychol. Rev. 50, 108-137 Dec.

Herniman, S.E., Cotton, S.M., Killackey, E., Hester, R., Allott, K.A., 2018. Co-morbid depressive disorder is associated with better neurocognitive performance in first episode schizophrenia spectrum. J. Affect. Disord. 229, 498-505.

Keefe, R.S.1., McClintock, S.M., Roth, R.M., Doraiswamy, P.M., Tiger, S., M.M., 2014 Cognitive effects of pharmacotherapy for major depressive disorder. J. Clin. Psychiatry 75, 864-876.

Kemp, R., David, A., 1996. Psychological predictors of insight and compliance in psychotic patients. Br. J. Psychiatry 169, 444-450.

Klaas, H.S., Clémence, A., Marion-Veyron, R., Antonietti, J.P., Alameda, L., Golay, P., Conus, P., 2017. Insight as a social identity process in the evolution of psychosocial functioning in the early phase of psychosis. Psychol. Med. 47, 718-729.

Lee, R.S.C., Hermens, D.F., Porter, M.A., Redoblado-Hodge, M.A., 2012. A meta-analysis of cognitive deficits in first-episode major depressive disorder. J. Affect. Disord. 140 (2), 113-124 Oct.

Lindgren, M., Mäntylä, T., Rikandi, E., Torniainen-Holm, M., Morales-Muñoz, I., Kieseppä, T., Mantere, O., Suvisaari, J., 2017. Childhood adversities and clinical symptomatology in first-episode psychosis. Psychiatry Res. 258, 374-381.

Lindgren, M., Torniainen-Holm, M., Heiskanen, I., Voutilainen, G., Pulkkinen, U., Mehtälä, T., Jokela, M., Kieseppä, T., Suvisaari, J., Therman, S., 2018. Theory of mind in a first-episode psychosis population using the hinting task. Psychiatry Res. 263, 185-192.

Lucas, S., Fitzgerald, D., Redoblado-Hodge, M.A., Anderson, J., Sanbrook, M., Harris, A., Brennan, J., 2004. Neuropsychological correlates of symptom profiles in first episode schizophrenia. Schizophr. Res. 71, 323-330.

Lysaker, P.H., Davis, L.W., Lightfoot, J., Hunter, N., Stasburger, A., 2005. Association of neurocognition, anxiety, positive and negative symptoms with coping preference in schizophrenia spectrum disorders. Schizophr. Res. 80, 163-171.

McEnery, C., Lim, M.H., Tremain, H., Knowles, A., Alvarez-Jimenez, M., 2019. Prevalence rate of social anxiety disorder in individuals with a psychotic disorder: a systematic review and meta-analysis. Schizophr. Res. 208, 25-33 Jun.

Mesholam-Gately, R.I., Giuliano, A.J., Goff, K.P., Faraone, S.V., Seidman, L.J., 2009. Neurocognition in first-episode schizophrenia: a meta-analytic review. Neuropsychology 23, 315-336.

Mollon, J., Reichenberg, A., 2018. Cognitive development prior to onset of psychosis. Psychol. Med. 48 (3), 392-403 Feb.

Morales-Muñoz, I., Suvisaari, J., Therman, S., Torniainen-Holm, M., Mäntylä, T., Rikandi, E., Mantere, O., Kieseppä, T., Lindgren, M., 2018. Childhood adversities and cognitive deficits in first-episode psychosis. Schizophr. Res. 197, 596-598 Jul.

Ohmuro, N., Matsumoto, K., Katsura, M., Obara, C., Kikuchi, T., Hamaie, Y., Sakuma, A., Iizuka, K., Ito, F., Matsuoka, H., 2015. The association between cognitive deficits and depressive symptoms in at-risk mental state: a comparison with first-episode psychosis. Schizophr. Res. 162, 67-73.

Potvin, O., Bergua, V., Meillon, C., Le Goff, M., Bouisson, J., Dartigues, J.F., Amieva, H., 2013. State anxiety and cognitive functioning in older adults. Am. J. Geriatr. Psychiatry 21, 915-924.

Rajji, T.K., Miranda, D., Mulsant, B.H., 2014. Cognition, function, and disability in patients with schizophrenia: a review of longitudinal studies. Can. J. Psychiatry 59 (1), 13-17 Jan.

Salthouse, T.A., 2012. How general are the effects of trait anxiety and depressive symptoms on cognitive functioning? Emotion 12, 1075-1084.

Sánchez-Torres, A.M., Moreno-Izco, L., Lorente-Omeñaca, R., Cabrera, B., Lobo, A., González-Pinto, A.M., Merchán-Naranjo, J., Corripio, I., Vieta, E., de la Serna, E., Butjosa, A., Contreras, F., Sarró, S., Mezquida, G., Ribeiro, M., Bernardo, M., Cuesta, M.J., PEPs group, 2018. Individual trajectories of cognitive performance in first episode psychosis: a 2-year follow-up study. Eur. Arch. Psychiatry Clin. Neurosci. 268, 699-711.

Sim, K., Chua, T.H., Chan, Y.H., Mahendran, R., Chong, S.A., 2006. Psychiatric comorbidity in first episode schizophrenia: a 2 year, longitudinal outcome study. J. Psychiatr. Res. 40, 656-663. 
Stouten, L.H., Veling, W., Laan, W., van der Helm, M., van der Gaag, M., 2017. Psychosocial functioning in first-episode psychosis and associations with neurocognition, social cognition, psychotic and affective symptoms. Early Interv. Psychiatry $11,23-36$

Upthegrove, R., Birchwood, M., Ross, K., Brunett, K., McCollum, R., Jones, L., 2010. The evolution of depression and suicidality in first episode psychosis. Acta Psychiatr. Scand. 122, 211-218.

Upthegrove, R., Ross, K., Brunet, K., McCollum, R., Jones, L., 2014. Depression in first episode psychosis: the role of subordination and shame. Psychiatry Res. 217, $177-184$.
Ventura, J., Green, M., Shaner, A., Liberman, R.P., 1993. Training and quality assurance on the BPRS: "the drift busters". Int. J. Methods Psychiatr. Res. 3, 221-224.

Vernon, J.A., Grudnikoff, E., Seidman, A.J., Frazier, T.W., Vemulapalli, M.S., Pareek, P., Goldberg, T.E., Kane, J.M., Correll, C.U., 2014. Antidepressants for cognitive impairment in schizophrenia - A systematic review and meta-analysis. Schizophr. Res. 159, 385-394.

Wang, M.Y., Ho, N.F., Sum, M.Y., Collinson, S.L., Sim, K., 2016. Impact of duration of untreated psychosis and premorbid intelligence on cognitive functioning in patients with first-episode schizophrenia. Schizophr. Res. 175, 97-102. 